\title{
Dental Enamel Microstructure Exposed to Non-Alcoholic Beverages In Vitro
}

\author{
A. Tanevitch $1 *$, A. Abal 1, G. Lazo1, C. Gigena 1 , M.J. Ingeniero1, A. Barceló1, E. Barrasa1, C. Ogas 1
}

1. Facultad de Odontología. Universidad Nacional de La Plata. La Plata, Argentina

* Corresponding author: atanevitch@gmail.com

The dental enamel demineralization phenomena by acids produce a tissue deterioration that expressed as erosion or caries lesions. These are not nutritional diseases, but related to diet and its chemical effects on teeth [1]. Erosion is the result of cumulative exposure to acids in times greater than 30 minutes [2]. Dental enamel consists of prisms that described as cylindrical rods, surrounded by sheaths and formed by a packing of hydroxyapatite crystals. Crystals with different angulation converge in the sheath, being an area that contains more enamel proteins than other regions. Mineralization of dental enamel decrease from external surface to dentine junction [3]. The parallel arrangement of the prisms in the external enamel zone forms the radial enamel, while the characteristic prism cross-linking of the internal zone configures the Hunter- Schreger bands. Our purpose was to describe the morphological aspect of the microstructure of tooth enamel exposed to different beverages. Also, analyse the chemical composition and $\mathrm{pH}$ of drinks.

Dental enamel samples were included in resin and then worn and polished longitudinally. Five commercial non-alcoholic drinks (two-flavoured orange water, a citrus flavour carbonated water, a cola and orange soft drink) and two prepared juices (orange and lemon flavour) were used. The immersion cycles were 3 minutes- 4 times per day- 14 days using artificial saliva as a conservation means between cycles. The artificial saliva composition is Potassium Chloride 0.06-Potassium Phosphate 0.17 g- Sodium Chloride 0.2 g- Calcium Chloride 0.148 g- Magnesium Chloride 0.025 g- CMC 5 g- Sorbitol 15 g - water csp 500 cc. The samples were metallized and observed at ESEM (SeMFi-LIMF-FI-UNLP). The morphological description was made considering external and internal enamel surface.

In the sections of healthy enamel worn and polished, the contours of the prisms were not very evident (Fig. 1A) Selective dissolution of the prism crystals determined morphological alterations in the microstructure of the enamel. The prisms could present the depressed centre and the conserved contours or the inverse pattern (Fig.1 B y C). The interprismatic region appeared wide and irregular (Fig 2). Artificial saliva caused the deposition of a thin and discontinuous film on the enamel surface (Fig. 2C). Acidifying additives such as citric acid, phosphoric acid, malic acid and EDTA, carbonated water, sodium polyphosphate, lemon and orange juice were found in drinks composition. In addition these contain sugars (JAF), carboxymethyl cellulose that acts as a thickener and film former, preservatives (CONS), vitamins, dyes (COL), caffeine All beverages studied had an acidic $\mathrm{pH}$ less than four. We conclude that all drinks produce enamel demineralization compatible with erosion lesions [4].

\section{References}

[1] T. Imfeld, Ther. Umsch. 65(2) (2008) p. 69.

[2] D.H.J. Jager, et al., J. Dentistry. 40(12) (2012) p. 1103

[3] M.A. El-Zainy, A.M. Halawa, A.A. Rabea, J. American Science. 8(3) (2012) p. 632

[4] Our acknowledgment to Microscopy and Microanalyses Service (LIMF-FI-UNLP) for their collaboration and technical support. 
A

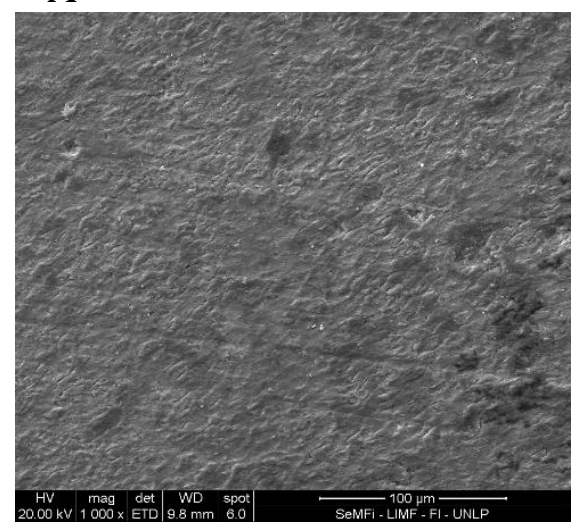

B

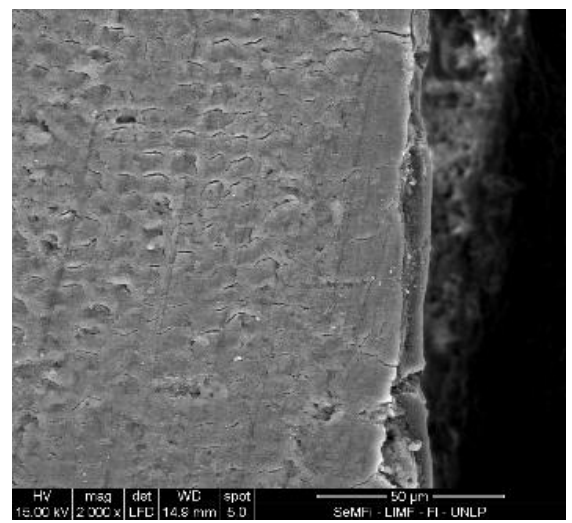

$\mathrm{C}$

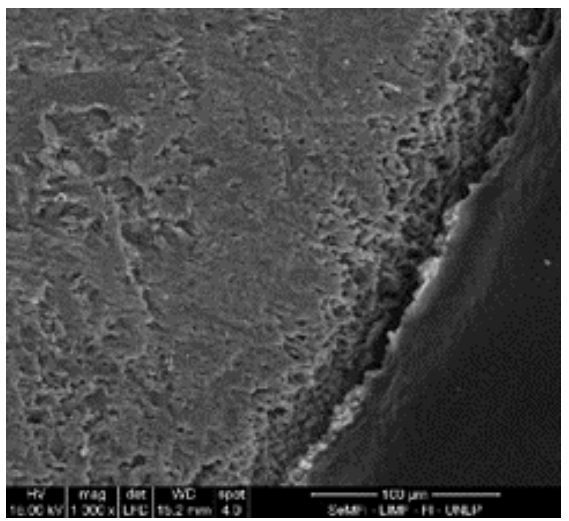

Figure 1. A: healthy enamel (ESEM x 1000); the contour of the prisms is not very evident. B and C: flavoured orange water. External enamel. B: prisms in cross section appeared with the centres depressed (ESEM x 2000), C: internal enamel, irregular morphology of the prisms (ESEM x 1000)

A

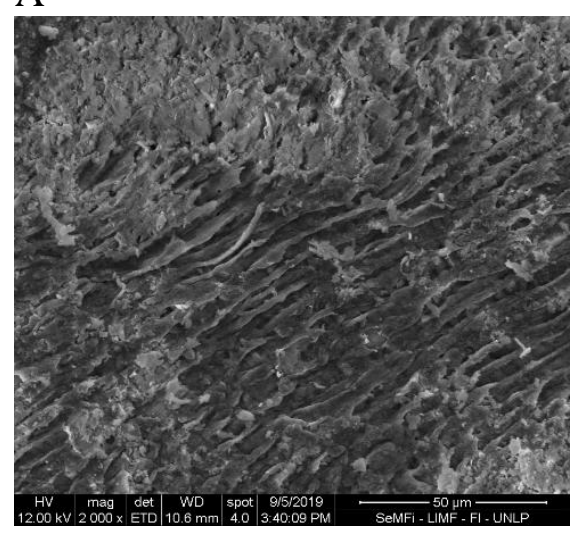

$\mathrm{D}$

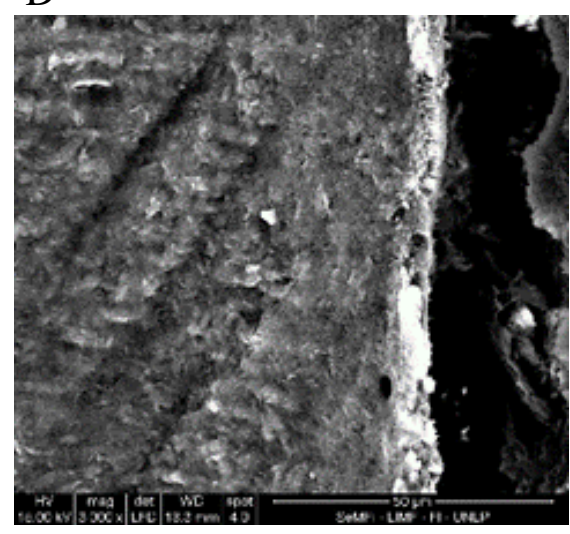

B

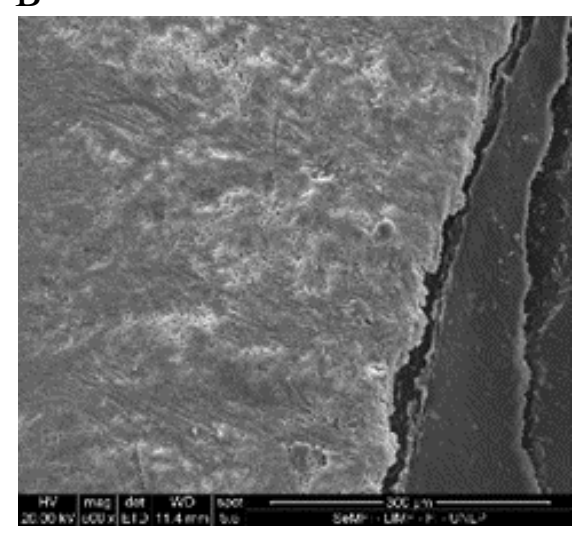

$\mathrm{E}$

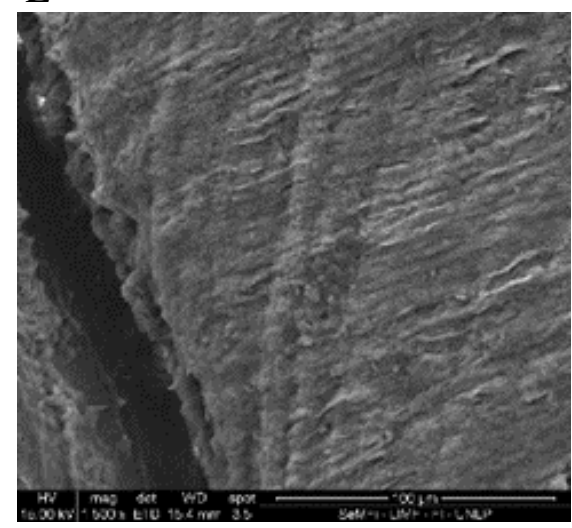

$\mathrm{C}$

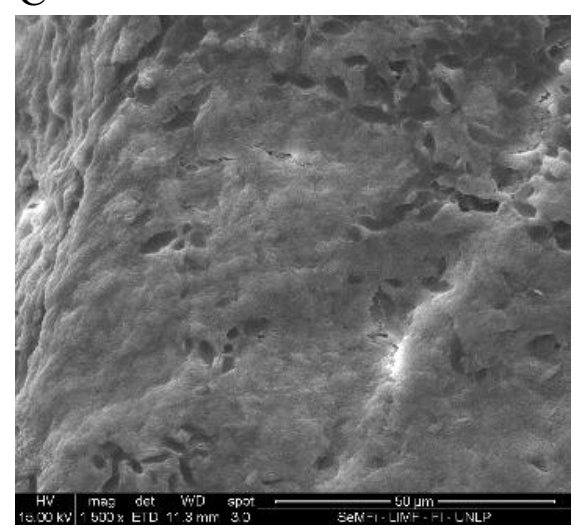

$\mathrm{F}$

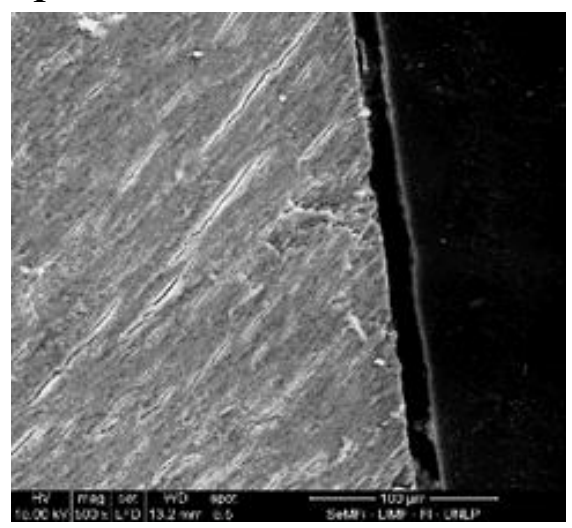

Figure 2. Micrograph of dental enamel after beverage action. A: citrus flavour carbonated water. The mineral loss affected rod outline (ESEM x 2000). B: carbonated orange drink. Internal enamel showed increased porosity (ESEM x 600). C: Artificial saliva caused a thin and irregular deposit of organic material on the enamel (ESEM x 500). D: lemon juice powder. Deep grooves between the rods observed (ESEM x 3000). E: carbonated cola drink. The Striae of Retzius manifested (ESEM x 500). F: orange juice powder. External enamel. The boundary between the rods was less obvious (ESEM x 500). 